\title{
Electrical Characteristics of Carbon Nanotube Devices Prepared with Single Oxidative Point Defects
}

\author{
Landon Prisbrey, ${ }^{\dagger}$ David Roundy, ${ }^{\dagger}$ Kerstin Blank, ${ }^{\ddagger}$ Leonard S. Fifield, ${ }^{\S}$ and Ethan D. Minot ${ }^{*,+}$ \\ ${ }^{\dagger}$ Department of Physics, Oregon State University, Corvallis, Oregon 97331-6507, United States \\ ${ }^{\ddagger}$ Institute for Molecules and Materials, Radboud University, Nijmegen, The Netherlands

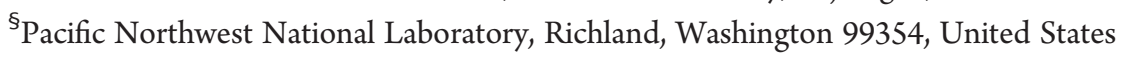

ABSTRACT: A thorough understanding of how electrons pass through point defects in carbon nanotubes is crucial for building carbon nanotube devices. We have generated point defects in the sidewalls of pristine carbon nanotubes via voltage pulses from a conducting atomic force microscope probe and studied the resulting changes in electron transport properties. We find that the incorporation of an oxidative defect leads to a variety of possible electrical signatures including sudden switching events, resonant scattering, and breaking of the symmetry between electron and hole

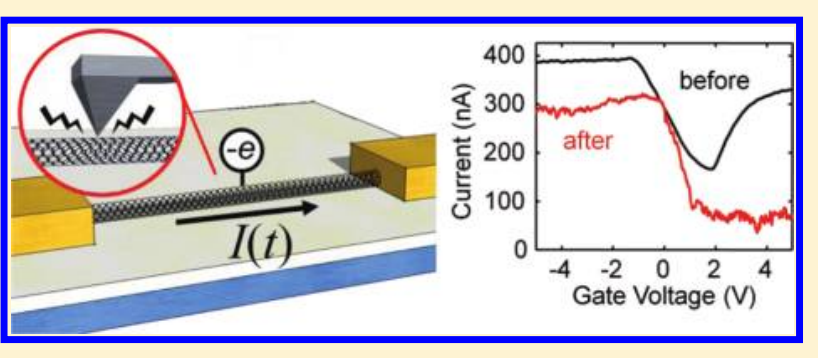
transport. We discuss the relationship between these different electronic signatures and the chemical structure/charge state of the defect. Tunneling through a defect-induced Coulomb barrier is modeled with numerical Verlet integration of Schrodinger's equation and compared with experimental results.

\section{INTRODUCTION}

Recently, much work has gone into utilizing the properties of defects in carbon nanotubes ( $\mathrm{CNTs}$ ). Devices with a single point defect have been used as single molecule sensors, ${ }^{1,2}$ and CNT network devices with multiple defects have shown higher sensitivity to certain chemicals compared to pristine CNT devices. ${ }^{3}$

It is an ongoing challenge to link theoretical and experimental studies of the electronic properties of CNT defects. Previous scanning tunneling microscope spectroscopy experiments have shown that defects can locally change the density of states of a CNT. ${ }^{4-6}$ Electron transport experiments on metallic CNTs with single defects have shown strongly suppressed conductance for electron doping, but much less suppression for hole doping. ${ }^{7}$ Theoretical work has focused on band structure calculations of CNTs with defects and often predicts the formation of localized impurity states in and around the band gap. ${ }^{8-14}$ A small number of theoretical studies have attempted to link defect structure to electron transport characteristics. 8,15

Oxidative defects in CNTs are typically introduced using gas phase or liquid phase treatments such as exposure to heat, ${ }^{16}$ ozone, ${ }^{17,18}$ acids, ${ }^{1,7,19,20}$ or peroxides. ${ }^{21,22}$ To generate individual oxidative point defects for single molecule sensing, ${ }^{1,2}$ additional techniques have been developed, including feedback-controlled electrochemical attack ${ }^{7}$ and local anodic oxidation utilizing voltage pulses from an $\mathrm{AFM}^{23,24}$ or $\mathrm{STM}^{25}$ probe. Nonoxidative point defects in CNTs have also been investigated, for example, through radical addition with diazonium compounds; ${ }^{26,27}$ however, nonoxidative point defects are not considered in our current work.

Depending on the oxidant and/or process, a variety of oxidative defects can be created in the sidewall of a CNT.
Possible defects include ethers/epoxides $(\mathrm{C}-\mathrm{O}-\mathrm{C})$, alcohols $(\mathrm{C}-\mathrm{OH})$, ketones/aldehydes $(\mathrm{C}=\mathrm{O})$, and carboxylic acids $(\mathrm{COOH}) .{ }^{28,29}$ Bulk chemistry methods yield mixtures of these different oxidation states. No bulk approach, nor any individualdefect approach, has been demonstrated to offer complete control over the chemical nature of the defect. An additional challenge is that traditional methods to identify the chemical structure of the defect, such as X-ray photoelectron spectroscopy, are not sensitive at the single-defect level. Only carboxylic acid groups have been previously identified at the single-defect level. ${ }^{1}$

We have used the AFM technique to create long-lived oxidative point defects in 15 different individually contacted quasi-metallic CNTs (small band gap CNTs) and observed a variety of different transport signatures. Half of these pointdefect devices showed strongly suppressed conductance for electron doping, but much less suppression for hole doping. We present a new model to explain this characteristic behavior. Our other point-defect devices showed electron transport characteristics that are consistent with resonant scattering from the discrete bound-state energy levels associated with the defect. Previous theoretical work has predicted this phenomenon, ${ }^{15}$ but it has not been previously observed in experiment. Overall, our results illustrate the wide range of electron transport characteristics that are associated with oxidative point defects in CNTs and suggest new ways to identify the chemical structure of point defects.

\footnotetext{
Received: September 14, 2011

Revised: December 19, 2011

Published: December 21, 2011
} 


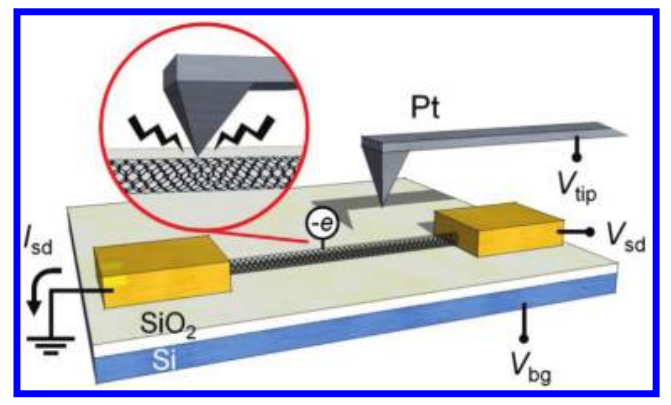

Figure 1. Schematic illustrating CNT FET device, the defect generation procedure, and the scanning gate microscopy technique. Not to scale.

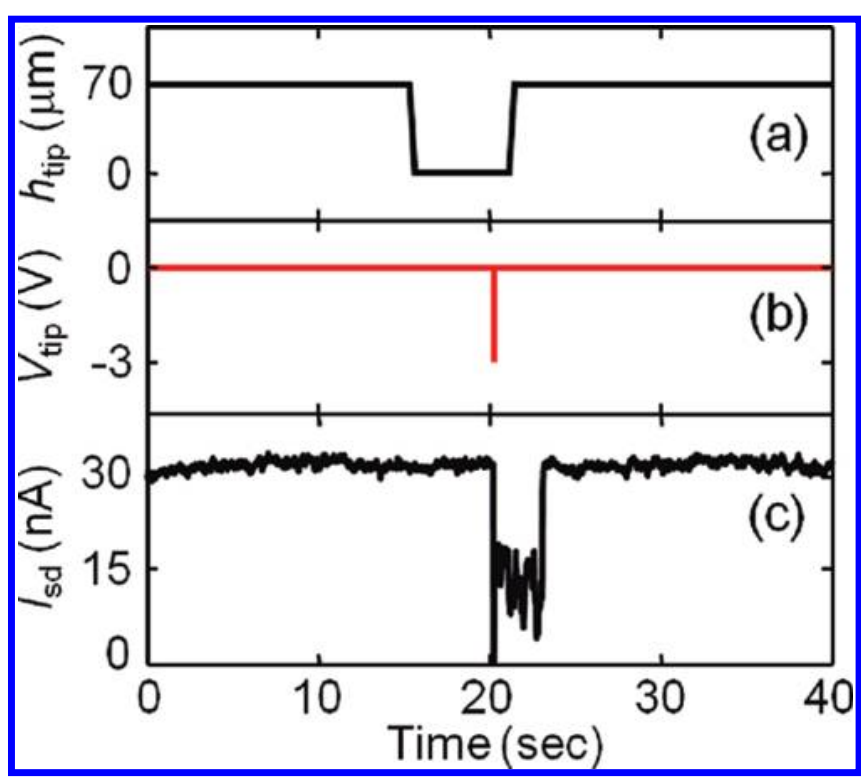

Figure 2. Creation of a short-lived CNT defect. The tip was lowered until it touched the CNT, a voltage pulse was applied between the AFM tip and the CNT, and the tip was retracted. The time traces show (a) tip height, $h_{\text {tip }}$, (b) tip voltage, $V_{\text {tip }}$, and (c) current through the CNT $\left(V_{\mathrm{sd}}=25 \mathrm{mV}\right)$.

\section{METHODS}

Individual CNT devices were made by patterning iron catalyst on a $\mathrm{SiO}_{2} / \mathrm{Si}$ substrate (300 nm oxide) and growing CNTs by catalyzed chemical vapor deposition $(\mathrm{CVD})^{30,31}$ (see ref 31 for growth parameters). Source and drain electrodes were patterned with photolithography to produce circuits with a number of CNTs connected in parallel. Electric force microscopy ${ }^{32}$ and scanning gate microscopy (SGM $)^{33}$ were used to identify circuits whose gate response is dominated by a single small band gap CNT (Figure 1). The CNT diameters ranged from 1 to $3 \mathrm{~nm}$. Previous authors have shown that the band gap of such quasimetallic CNTs scales inversely with diameter and arises mainly from strong electron-electron interactions (Mott gaps of 20$60 \mathrm{meV}) .^{34}$ Small band gap CNTs are the most promising for single-molecule sensors; therefore, we have focused on small band gap CNTs. Studies of oxidative point defects in large band gap CNTs would be interesting but have not yet been undertaken.

Point defects were generated in ambient conditions with voltage pulses from a Pt-coated AFM probe. ${ }^{23,35}$ A home-built AFM stage with electrical probes was used to contact the CNT

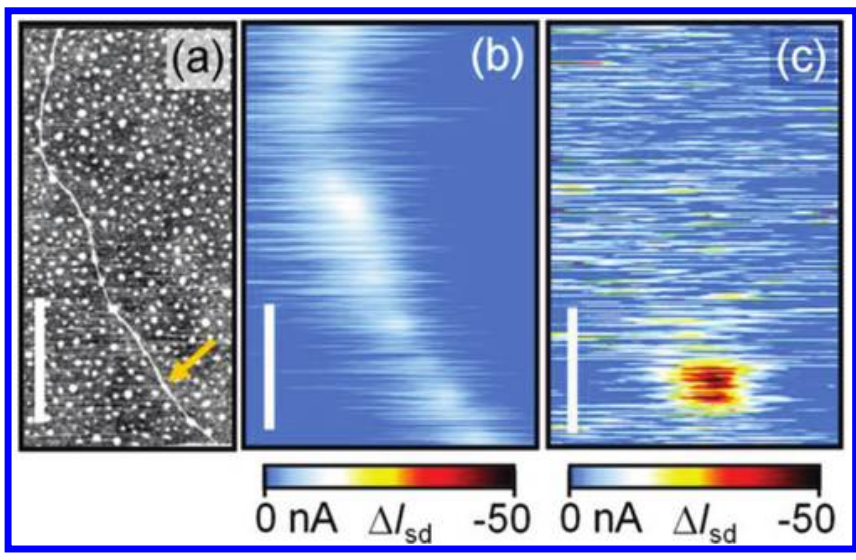

Figure 3. (a) AFM topography. A defect was created in the CNT where indicated by the arrow. Dots on the $\mathrm{SiO}_{2}$ surface are residue left over from the process of fabricating the metal electrodes. (b, c) Scanning gate microscopy images before and after defect generation. Imaging parameters are $V_{\mathrm{sd}}=50 \mathrm{mV}, V_{\mathrm{bg}}=-700 \mathrm{mV}, V_{\text {tip }}=3 \mathrm{~V}$, and height $=20 \mathrm{~nm}$. To calculate $\Delta I_{\mathrm{sd}}$, we first compute $\left\langle I_{\mathrm{sd}}\right\rangle_{\text {line }}$ (average current during each line trace) and then plot the difference $\Delta I_{\text {sd }}(x, y)=I_{\text {sd }}(x, y)-\left\langle I_{\text {sd }}\right\rangle_{\text {line. }}$. This is done to compensate for low-frequency fluctuations in $I_{\mathrm{sd}}(t)$. The line averaged current is $\left\langle I_{\text {sd }}\right\rangle_{\text {line }} \sim 330 \mathrm{nA}$ in panel $\mathrm{b}$ and $\left\langle I_{\mathrm{sd}}\right\rangle_{\text {line }} \sim 170 \mathrm{nA}$ in panel c. Scale bars: $1 \mu \mathrm{m}$.

device. A small source-drain bias $\left(V_{\mathrm{sd}}=25 \mathrm{mV}\right)$ was applied to monitor the conductance of the device before, during, and after modification. To create a defect, we lowered the AFM probe onto the CNT (contact force $=6-9 \mathrm{nN}$ ) and applied a $-3 \mathrm{~V}$, $15 \mathrm{~ms}$ square wave pulse to the tip.

\section{RESULTS AND DISCUSSION}

Successful defect creation is manifested as a drop in CNT conductance. Afterward, the CNT may remain permanently modified or else "self-heal", which we observed as an abrupt return of the conductance to the premodified state.

We first discuss the self-healing phenomenon. Figure 2 shows electrical current through a CNT before, during, and after defect creation $\left(V_{\mathrm{sd}}=25 \mathrm{mV}\right.$ throughout the experiment). When a voltage pulse was applied from an AFM tip at $t=20 \mathrm{~s}$, a sudden increase in resistance was observed (a drop in current). The device remained modified for $3 \mathrm{~s}$ before the resistance returned to the premodified state. We note that the self-healing event does not coincide with lifting the AFM tip away from the surface. Such short-lived defects were observed in four devices with lifetimes ranging between 3 and $30 \mathrm{~s}$ and defect resistances ranging between $5 \mathrm{k} \Omega$ and $1 \mathrm{M} \Omega$.

It is reasonable to assume that a short-lived defect is chemically unstable and/or energetically unfavorable. For example, the following two mechanisms might be considered. The voltage pulse may lead to $\mathrm{C}-\mathrm{C}$ bond rotation that is reversible with fast time scales at room temperature. ${ }^{25}$ Alternatively, oxygen might be added to the nanotube in a high-energy configuration, leading to structural rearrangements and subsequent elimination of the oxygen. ${ }^{8}$ Although not clearly defined chemically, our current results show that these unstable states can be observed by transport measurements at the single defect level.

Unstable defects are not relevant for the development of CNT electronics applications, and we now focus on CNTs with oxidative defects that were long-lived. Scanning gate microscopy measurements, where a biased AFM tip operates as a roaming local gate 


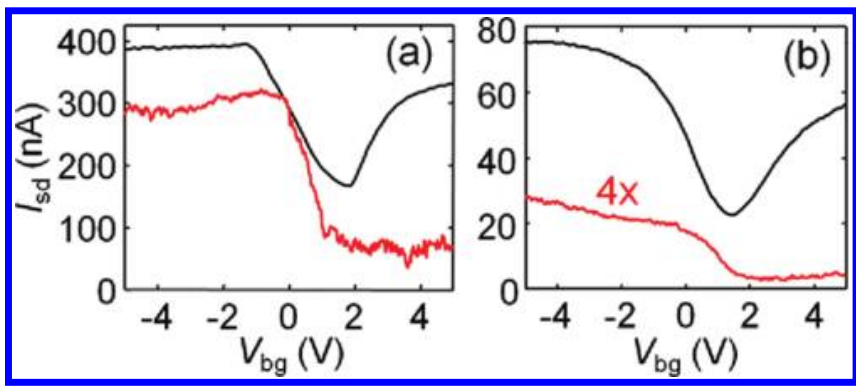

Figure 4. Transistor response of two CNT FET devices before (black) and after (red) defect creation. The red curves are the average of a few $I_{\mathrm{sd}}\left(V_{\mathrm{bg}}\right)$ sweeps. In panel b, the red curve is multiplied by $4\left(V_{\mathrm{sd}}=25 \mathrm{mV}\right)$.

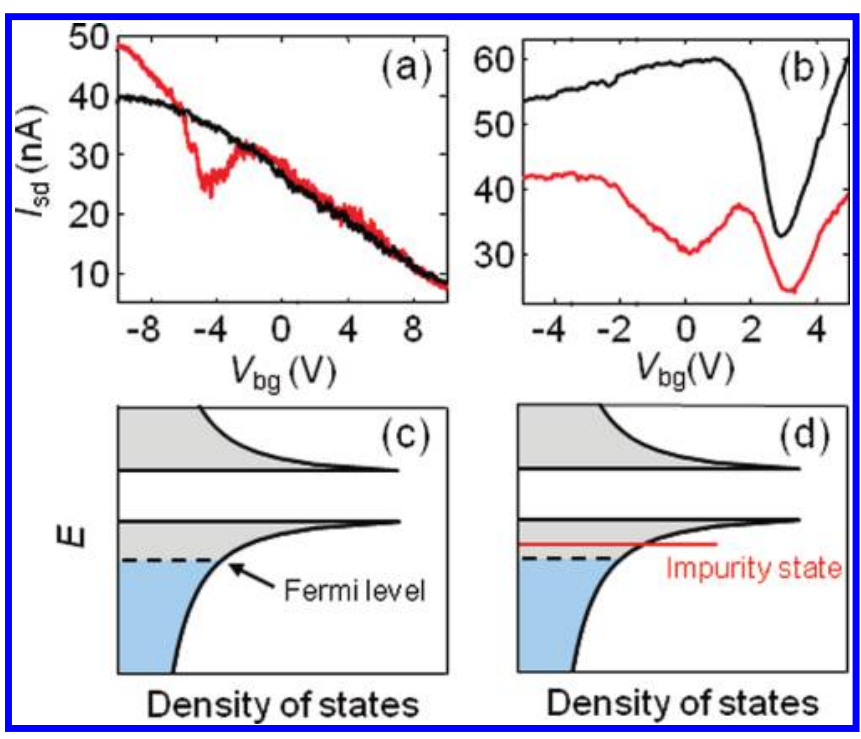

Figure 5. Resonant scattering behavior. (a, b) Transistor characteristics of two CNT devices before (black) and after (red) defect creation $\left(V_{\text {sd }}=\right.$ $25 \mathrm{mV})$. The red curves are the average of a few $I_{\mathrm{sd}}\left(V_{\mathrm{bg}}\right)$ sweeps. (c) Density of states of a pristine CNT. (d) Local density of states of a CNT around a neutral defect.

(Figure 1), were performed before and after stable defect creation to confirm that electrical transport is dominated by the introduced defect. Shown in Figure 3a is a topography scan of a pristine CNT. SGM measurements show that it is gate sensitive along its entire length (Figure 3b). An identical measurement following defect creation reveals that gate sensitivity is localized to the region around the defect, and this region is behaving as a bottleneck for charge transport (Figure 3c). Control experiments show that the observed effects were not caused by local charge injection into the oxide. ${ }^{36}$ Voltage pulses identical to those which produce defects were applied between the AFM tip and the oxide near the CNT (within 10-500 nm) with no observable effect on conductance.

Stable-defect device characteristics are shown in Figures 4, 5, and 6. Each figure shows before-defect and after-defect low-bias transport characteristics representing the range of behavior that we have observed from quasi-metallic CNTs.

Figure 4 shows two devices for which the overall conductance decreased and n-type conductance was suppressed relative to p-type conductance (i.e., conductance at positive gate voltages was suppressed relative to the conductance at negative gate voltages). We also observed a notable increase in conductance

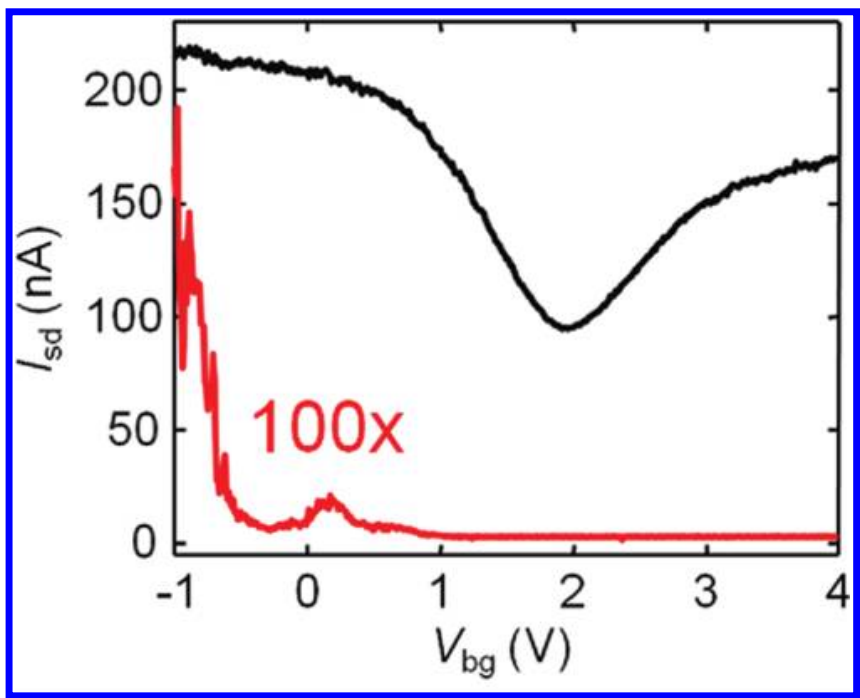

Figure 6. Transistor characteristics of a CNT FET device before (black) and after (red) defect creation. The red curve is multiplied by $100\left(V_{\text {sd }}=25 \mathrm{mV}\right)$.

fluctuations after incorporation of the defect. Of the 15 defect devices we have characterized, eight were qualitatively similar to Figure 4. The diameters of these CNTs were measured using tapping mode AFM imaging and range between 1.3 and $3.1 \mathrm{~nm}$. We note that these AFM measurements were done with standard imaging parameters (free air amplitude $\sim 100 \mathrm{~nm}$ ) and therefore systematically underestimated the CNT diameters due to the tapping force. ${ }^{37}$

Figure 5 shows two devices where the point defect modification resulted in a minimal conductance change at most gate voltages but caused sharp conductance dips at specific gate voltages. In addition, when $V_{\mathrm{bg}}$ was in the vicinity of one of these dips, we sometimes observed random telegraph noise. ${ }^{38}$ Five out of 15 devices were qualitatively similar to Figure 5 . The diameters of these CNTs range from 1.1 to $2.5 \mathrm{~nm}$.

Lastly, Figure 6 shows a device where n-type conductance is heavily suppressed and a sharp peak emerges in the $I_{\mathrm{sd}}\left(V_{\mathrm{bg}}\right)$ characteristics. Two out of the 15 devices that we prepared with point defects showed this behavior. Two additional CNT devices, in which we did not intentionally create defects, showed similar electrical characteristics. The observation of defect-like behavior in unmodified CNTs indicates that electrically disruptive point defects do occur naturally but are relatively rare in our CVD-grown CNTs. The diameters of these CNTs range from 1.6 to $3.4 \mathrm{~nm}$.

We first discuss the breaking of the symmetry between electron transport and hole transport that is seen in Figure 4. Suppression of n-type conductance has been observed previously, ${ }^{1,7}$ and theoretical explanations have been sought. ${ }^{8}$ Ashraf et al. performed band structure calculations of CNTs containing clusters of covalently bound oxygen defects. ${ }^{8}$ These calculations predict that certain cluster arrangements of multiple defects can suppress n-type conductance by a resonant scattering mechanism. However, this proposed mechanism does not explain the ubiquitous nature of suppressed n-type conductance that we observe.

We hypothesize that suppression of n-type conductance is related to the negative charge of oxidative point defects. Oxygen is more electronegative than carbon, and therefore, all oxidative defects carry some negative charge. The point defect structure 


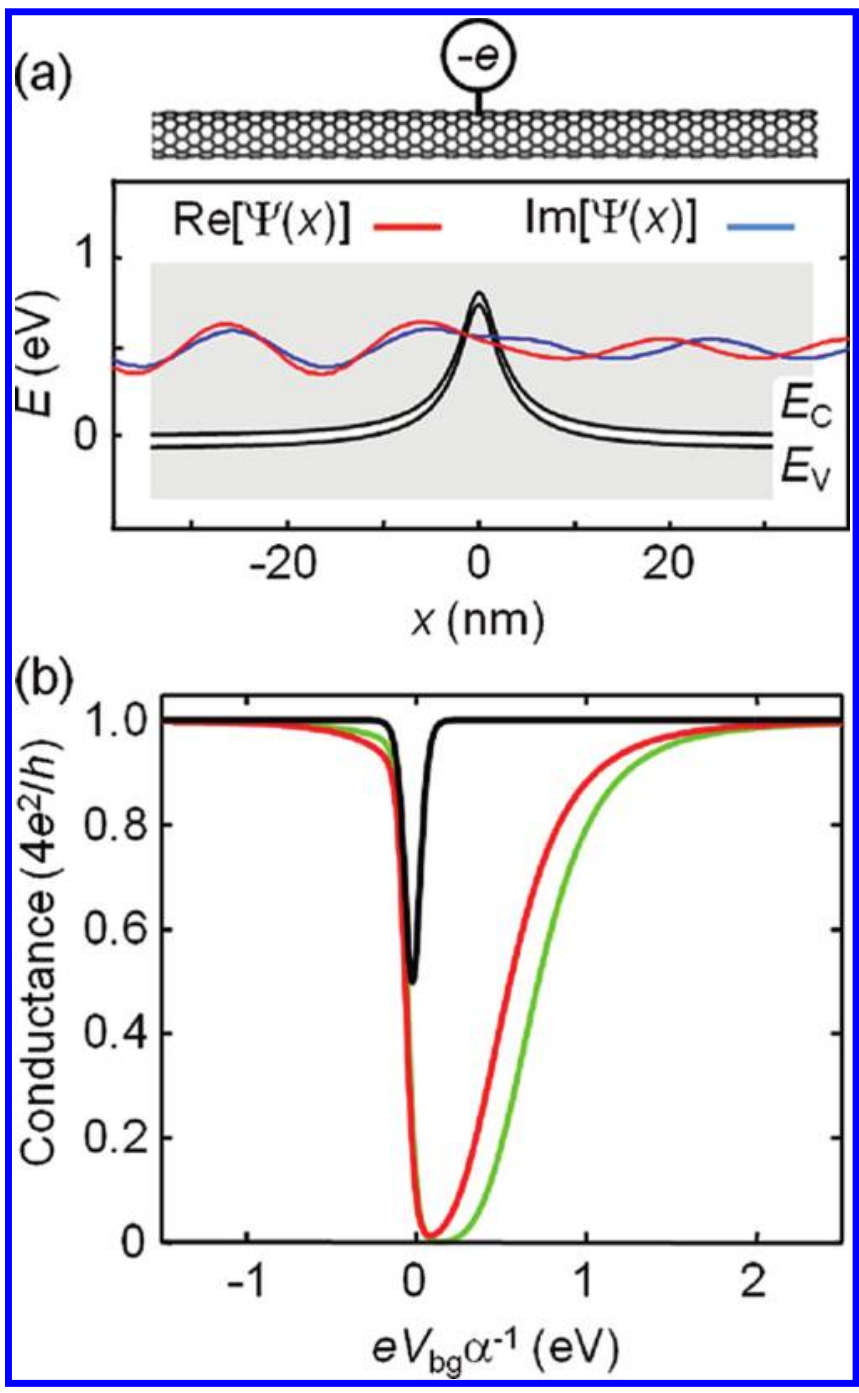

Figure 7. Theoretical results. (a) A typical electron wave function, $\Psi(x)$, tunneling through a Coulomb barrier formed by a charged defect in a small band gap CNT. The real and imaginary parts of the wave function are shown. The conduction and valence band edges are indicated by $E_{\mathrm{C}}$ and $E_{\mathrm{V}}$, respectively. (b) Conductance with and without a Coulomb barrier assuming perfect transmission through the rest of the device. The Fermi level in the CNT is set by $e V_{\mathrm{bg}} \alpha^{-1}$, where $\alpha$ is the gate coupling constant. The black curve is calculated without a Coulomb barrier (no band bending), and the red and green curves correspond to Coulomb barriers with screening lengths of $8 \mathrm{~nm}$ and $1 \mu \mathrm{m}$, respectively. The calculation assumes electron effective mass $=m_{\mathrm{e}} / 150$, CNT band gap $=60 \mathrm{meV}, k_{\mathrm{B}} T=25 \mathrm{meV}$, defect charge $=-e$, and position of charge $=1.5 \mathrm{~nm}$ above the CNT axis.

with highest charge is likely a deprotonated carboxyl group $\left(\mathrm{COO}^{-}\right)$, which is charged to $-e$ (the charge of an electron). This state occurs in aqueous environments when the $\mathrm{pH}$ of the solution is higher than the $\mathrm{p} K_{\mathrm{a}}$ value of the carboxyl group. Under ambient conditions a thin layer of water on the surface may facilitate deprotonation. The Coulomb potential from a negative charge will be a barrier to electron transport in small bandgap CNTs but not to hole transport.

To test the importance of the defect charge, we model the electrostatic potential near the defect as a screened Coulomb potential. Figure $7 \mathrm{a}$ illustrates the specific case of a CNT with a band gap of $60 \mathrm{meV}$ and a charge $-e$ placed $z=1.5 \mathrm{~nm}$ from the long axis of the CNT such that the potential along the CNT axis is given by

$$
V(x)=\frac{1}{4 \pi \varepsilon_{0}} \frac{-e}{\sqrt{x^{2}+z^{2}}} e^{-\sqrt{x^{2}+z^{2}} / \lambda}
$$

where $\lambda$ is the electrostatic screening length set by the environment.

For the electrostatic potential shown in Figure 7a, we computed the electron transmission probability at various energies, $T(E)$, using numerical Verlet integration ${ }^{39}$ of Schrödinger's equation. In this technique a plane wave solution is assumed for the transmitted wave, and the remainder of the function is numerically constructed from the second derivative available from the Schrödinger equation. We use an electron effective mass of $m_{\mathrm{e}} / 150$, consistent with the dispersion relation of a $\mathrm{CNT}$ with a $60 \mathrm{meV}$ bandgap. We make the simplifying assumption that interband transitions do not occur. A more complete model would account for the finite probability of interband transitions and the CNT relativistic dispersion relation using a Dirac Hamiltonian formalism. Figure 7a shows a typical electron wave function tunneling through the barrier from left to right. The standing wave on the left side of the barrier is a result of interfering incident and reflected waves.

Figure $7 \mathrm{~b}$ shows the predicted conductance of the defect calculated from $T(E)$. Conductance as a function of gate voltage for a pair of $1 \mathrm{D}$ channels is calculated from the LandauerButtiker formalism

$$
I=\frac{4 e}{h} \int_{-\infty}^{\infty} T(E)\left[f\left(E, V_{\mathrm{bg}} \alpha^{-1}+V_{\mathrm{sd}}\right)-f\left(E, V_{\mathrm{bg}} \alpha^{-1}\right)\right] \mathrm{d} E
$$

where $e$ is the elementary charge, $h$ is the Planck constant, $f$ is the Fermi-Dirac distribution function at room temperature, $\alpha$ is the gate coupling constant, and $e V_{\mathrm{bg}} \alpha^{-1}$ and $e V_{\mathrm{bg}} \alpha^{-1}+e V_{\text {sd }}$ set the chemical potential for the left moving electrons and right moving electrons, respectively. The model predicts ambipolar behavior (symmetrical conductance for hole transport and electron transport) for the pristine CNT but suppressed conductance for electron transport when the Coulomb barrier is present.

The model shown in Figure 7 ignores several sources of resistance that will add in series with the resistance of a point defect. First, the contact resistance between the metal electrodes and the CNT adds resistance $\sim 100 \mathrm{k} \Omega$. Second, phonon scattering in the unmodified sections of CNT adds resistance $\sim 10 \mathrm{k} \Omega \mu \mathrm{m}^{-1}$. $^{40}$ Third, substrate-induced electrostatic disorder along the length of a pristine CNT broadens the "OFF state" in measurements of $I_{\mathrm{sd}}$ vs $V_{\mathrm{bg} .}{ }^{33}$ While all sources of resistance must be considered to explain the experimental $I_{\mathrm{sd}}\left(V_{\mathrm{bg}}\right)$ measurements, Figure 7 successfully predicts a significant increase in local resistance when a single charge is placed near a small band gap CNT. The model also successfully explains broken symmetry between electron transport and hole transport.

We next consider the sharp dips and peaks in the transport characteristics shown in Figures 5 and 6, which we hypothesize are a result of the localized electronic states associated with a defect. Band structure calculations for CNTs with point defects predict the formation of localized impurity states. ${ }^{8-14}$ These states have been experimentally observed in the local density of states around CNT defects, as measured with a scanning tunneling microscope. ${ }^{25,41-43}$ Theoretical work has predicted that such impurity states will cause resonant scattering in electron transport measurements. ${ }^{8,15}$ As the gate voltage modulates the Fermi level in a CNT, charge carriers 
may become resonant with impurity states as illustrated in Figure 5c, d. Resonant scattering behavior is expected if the Fermi level aligns with an impurity level that is within the conduction or valence band. Resonant scattering is a likely explanation for the sharp dips in conductance seen in Figure 5. Alternatively, we expect resonant tunneling behavior (sharp peaks in the conductance) if there is a Coulomb barrier impeding electron transport and the Fermi level aligns with an impurity state in the band gap. Resonant tunneling is a possible explanation for the apparent peaks seen in Figure 6.

The energy level structure of impurity states associated with a CNT defect depends on the chiral index of the CNT and the chemical composition of the defect. Because of these unknowns, we cannot directly compare our experimental results to theory. However, we expect that further theoretical and experimental work will lead to the development of low-bias electron transport as a spectroscopic tool for identifying the chemical nature of oxidative point defects in CNTs.

\section{CONCLUSION}

We conclude that the AFM method of incorporating point defects in CNTs leads to a range of different defect types with distinct electrical characteristics. Our modeling of electron transport through a Coulomb barrier shows that the charge of the defect, which varies based on chemical structure, strongly influences electrical characteristics. Our measurements of resonant scattering and tunneling suggest that the spectrum of impurity state energy levels associated with a defect also strongly influences electrical characteristics. Future work will aim for greater control over the defect generation process and more detailed theory to match the chemical structure of a defect with its electron transport signature.

\section{AUTHOR INFORMATION}

\section{Corresponding Author}

*E-mail: minote@science.oregonstate.edu.

\section{ACKNOWLEDGMENT}

We thank Kristina Prisbrey for assistance with Figure 1. We thank Paul Schuele and Sharp Laboratoriesfor assistance with device fabrication. The Pacific Northwest National Laboratory is operated by Battelle Memorial Institute for the U.S. Department of Energy. This work is funded by the Human Frontier Science Program. We thank the Oregon Nanoscience and Microtechnology Institute for support of preliminary experiments.

\section{REFERENCES}

(1) Goldsmith, B. R.; Coroneus, J. G.; Kane, A. A.; Weiss, G. A.; Collins, P. G. Nano Lett. 2008, 8, 189-194.

(2) Sorgenfrei, S.; Chiu, C.-y.; Gonzalez, R. L.; Yu, Y.-J.; Kim, P.; Nuckolls, C.; Shepard, K. L. Nature Nanotechnol. 2011, 6, 126-132.

(3) Robinson, J. A.; Snow, E. S.; Bădescu, Ș. C.; Reinecke, T. L.; Perkins, F. K. Nano Lett. 2006, 6, 1747-1751.

(4) Buchs, G.; Ruffieux, P.; Gröning, P.; Gröning, O. Appl. Phvs. Lett. 2008, 93, 073115.

(5) Tolvanen, A.; Buchs, G.; Ruffieux, P.; Gröning, P.; Gröning, O.; Krasheninnikov, A. V. Phys. Rev. B 2009, 79, 125430.

(6) Buchs, G.; Ruffieux, P.; Gröning, P.; Gröning, O. Appl. Phys. Lett. 2007, 90, 013104.

(7) Goldsmith, B. R.; Coroneus, J. G.; Khalap, V. R.; Kane, A. A.; Weiss, G. A.; Collins, P. G. Science 2007, 315, 77-81.

(8) Ashraf, M. K.; Bruque, N. A.; Pandey, R. R.; Collins, P. G.; Lake, R. K. Phys. Rev. B 2009, 79.
(9) da Silva, L. B.; Fagan, S. B.; Mota, R. I. Phvs. Chem. C 2009, 113, 8959-8963.

(10) Carneiro, M. A.; Venezuela, P.; Fagan, S. B. L. Phys. Chem. C 2008, 112, 14812-14815.

(11) Rocha, A. R.; Padilha, J. E.; Fazzio, A.; da Silva, A. J. R. Phvs. Rev. $\underline{\underline{B}}$ 2008, 77, 153406.

(12) Zhao, J.; Park, H.; Han, J.; Lu, J. P. I. Phvs. Chem. B 2004, $108,4227-4230$.

(13) Zhao, J.; Chen, Z.; Zhou, Z.; Park, H.; Schleyer, P.; von, R.; Lu, J. P. ChemPhvsChem 2005, 6, 598-601.

(14) Veloso, M. V.; Souza Filho, A. G.; Mendes Filho, J.; Fagan, S. B.; Mota, R. Chem. Phys. Lett. 2006, 430, 71-74.

(15) Choi, H. J.; Ihm, J.; Louie, S. G.; Cohen, M. L. Phvs. Rev. Lett. 2000, 84, 2917.

(16) Hou, P.-X.; Liu, C.; Cheng, H.-M. Carbon 2008, 46, 2003-2025.

(17) Mawhinney, D. B.; Naumenko, V.; Kuznetsova, A.; Yates, J. T.; Liu, J.; Smalley, R. E. I. Am. Chem. Soc. 2000, 122, 2383-2384.

(18) Banerjee, S.; Wong, S. S. I. Phvs. Chem. B 2002, 106, 12144-12151.

(19) Coroneus, J. G.; Goldsmith, B. R.; Lamboy, J. A.; Kane, A. A.; Collins, P. G.; Weiss, G. A. ChemPhysChem 2008, 9, 1053-1056.

(20) Kanai, Y.; Khalap, V. R.; Collins, P. G.; Grossman, J. C. Phys. Rev. Lett. 2010, 104, 066401.

(21) Miyata, Y.; Maniwa, Y.; Kataura, H. L.Phvs. Chem. B 2006, 110, 25 -29.

(22) Peng, Y.; Liu, H. Ind. Eng. Chem. Res. 2006, 45, 6483-6488.

(23) Park, J.-Y. Appl. Phvs. Lett. 2007, 90, 023112-3.

(24) Park, J.-Y.; Yaish, Y.; Brink, M.; Rosenblatt, S.; McEuen, P. L. Appl. Phvs. Lett. 2002, 80, 4446-4448.

(25) Berthe, M.; Yoshida, S.; Ebine, Y.; Kanazawa, K.; Okada, A.; Taninaka, A.; Takeuchi, O.; Fukui, N.; Shinohara, H.; Suzuki, S.; Sumitomo, K.; Kobayashi, Y.; Grandidier, B.; Stievenard, D.; Shigekawa, H. Nano Lett. 2007, 7, 3623-3627.

(26) Strano, M. S.; Dyke, C. A.; Usrey, M. L.; Barone, P. W.; Allen, M. J.; Shan, H.; Kittrell, C.; Hauge, R. H.; Tour, J. M.; Smalley, R. E. Science 2003, 301, 1519-1522.

(27) Goldsmith, B. R.; Mitala, J. J.; Josue, J.; Castro, A.; Lerner, M. B.; Bayburt, T. H.; Khamis, S. M.; Jones, R. A.; Brand, J. G.; Sligar, S. G.; Luetje, C. W.; Gelperin, A.; Rhodes, P. A.; Discher, B. M.; Johnson, A. T. C. ACS Nano 2011, 5, 5408-5416.

(28) Kundu, S.; Wang, Y.; Xia, W.; Muhler, M. I.Phvs. Chem.C 2008, 112, 16869-16878.

(29) Wepasnick, K. A.; Smith, B. A.; Bitter, J. L.; Howard Fairbrother, D. Anal. Bioanal. Chem. 2010, 396, 1003-1014.

(30) Kong, J.; Soh, H. T.; Cassell, A. M.; Quate, C. F.; Dai, H. Nature 1998, 395, 878-881.

(31) Leyden, M. R.; Schuman, C.; Sharf, T.; Kevek, J.; Remcho, V. T.; Minot, E. D. Proc. SPIE 2010, 77790H-77790H-11.

(32) Leng, Y. Proc. SPIE 1993, 35-39.

(33) Tans, S. J.; Dekker, C. Nature 2000, 404, 834-835.

(34) Deshpande, V. V.; Chandra, B.; Caldwell, R.; Novikov, D. S.; Hone, J.; Bockrath, M. Science 2009, 323, 106-110.

(35) Kim, D.-H.; Koo, J.-Y.; Kim, I.-I. Phvs. Rev. B 2003, 68, 113406.

(36) Prisbrey, L.; DeBorde, T.; Park, J.-Y.; Minot, E. D. Appl. Phvs. Lett. 2011, 99, 053125.

(37) DeBorde, T.; Joiner, J. C.; Leyden, M. R.; Minot, E. D. Nano Lett. 2008, 8, 3568-3571.

(38) Liu, F.; Bao, M.; Kim, H.-jun; Wang, K. L.; Li, C.; Liu, X.; Zhou, C. Appl. Phvs. Lett. 2005, 86, 163102.

(39) Thijssen, J. M. Computational Physics; Cambridge University Press: New York, 2007.

(40) Zhou, X.; Park, J.-Y.; Huang, S.; Liu, J.; McEuen, P. L. Phys. Rev. Lett. 2005, 95, 146805.

(41) Buchs, G.; Krasheninnikov, A. V.; Ruffieux, P.; Gröning, P.; Foster, A. S.; Nieminen, R. M.; Gröning, O. New J. Phys. 2007, 9, 275-275.

(42) Kim, H.; Lee, J.; Lee, S.; Kuk, Y.; Park, J.-Y.; Kahng, S.-I. Phvs. Rev. B 2005, 71, 235402.

(43) Lee, S.; Kim, G.; Kim, H.; Choi, B.-Y.; Lee, J.; Jeong, B. W.; Ihm, J.; Kuk, Y.; Kahng, S.-I. Phvs. Rev. Lett. 2005, 95, 166402. 